\title{
Serum luteinizing hormone level and luteinizing hormone/follicle-stimulating hormone ratio but not serum anti-Müllerian hormone level is related to ovarian volume in Korean women with polycystic ovary syndrome
}

\author{
Sungwook Chun \\ Department of Obstetrics and Gynecology, Inje University Haeundae Paik Hospital, Inje University College of Medicine, Busan, Korea
}

Objective: The aim of the present study was to investigate the relationship between ovarian follicle count and volume on ultrasonography and serum hormone levels including the levels of the anti-Müllerian hormone (AMH) and gonadotropin in women with the polycystic ovary syndrome (PCOS).

Methods: A total of 118 Korean women aged 18-35 years who were newly diagnosed with PCOS at a university hospital were included in this study. Serum LH, FSH, and AMH levels were measured in the early follicular phase, and the total antral follicle count (TFC) and the total ovarian volume (TOV) were assessed by ultrasonography. The correlations between serum hormonal parameters and ultrasonography characteristics in women with PCOS were evaluated using Pearson's correlation coefficients and a linear regression analysis.

Results: Serum AMH levels were significantly correlated with serum LH levels and LH/FSH ratios, and TFC and TOV were significantly correlated with each other on ultrasonography. Serum AMH and LH levels and the LH/FSH ratio were significantly correlated with TFC. Statistically significant correlations between TOV and the LH level $(r=0.208, p=0.024)$ and the LH/FSH ratio $(r=0.237, p=0.010)$ were observed. However, the serum AMH level was not significantly correlated with the ovarian volume, and this result did not change after adjusting for age and body mass index.

Conclusion: Serum AMH is not related to the ovarian volume in women with PCOS. My results suggest that serum LH level and the LH/FSH ratio may be more useful than the serum AMH level for representing the status of the ovarian volume in women with PCOS.

Keywords: Anti-Müllerian hormone; Luteinizing hormone; Luteinizing hormone/follicle-stimulating hormone ratio; Ovarian volume; Polycystic ovary syndrome

\section{Introduction}

Polycystic ovary syndrome (PCOS) is a common endocrine disorder,

Received: Apr 18, 2014 · Revised: May 20, 2014 · Accepted: Jun 2, 2014 Corresponding author: Sungwook Chun

Department of Obstetrics and Gynecology, Inje University Haeundae Paik Hospital, Inje University College of Medicine, 875 Haeun-daero, Haeundae-gu, Busan 612-030, Korea

Tel:+82-51-797-2020 Fax:+82-51-797-2030 E-mail:wooki1974@empal.com

This is an Open Access article distributed under the terms of the Creative Commons Attribution Non-Commercial License (http://creativecommons.org/licenses/by-nc/3.0/) which permits unrestricted non-commercial use, distribution, and reproduction in any medium, provided the original work is properly cited. affecting up to $5 \%$ to $10 \%$ of women of reproductive age worldwide [1]. If the current diagnostic criteria are used [2], the presence of two of three of the following criteria is sufficient to diagnose PCOS: oligoor anovulation, clinical and/or biochemical signs of hyperandrogenism, and/or ultrasonographic evidence of polycystic ovaries (PCO) after exclusion of other etiologies.

Increased antral follicle count (AFC) and increased ovarian size on ultrasonography are two cardinal features for the diagnosis of PCO. The ultrasonographic criteria for $\mathrm{PCO}$ requires the presence of $\geq 12$ follicles in either ovary measuring 2-9 $\mathrm{mm}$ in diameter and/or increased ovarian volume $\left(>10 \mathrm{~cm}^{3}\right)[2,3]$. Women with PCOS are likely 
to have not only a higher AFC but also a greater ovarian volume $[4,5]$. Inappropriate gonadotropin secretion and increased serum antiMüllerian hormone (AMH) levels are the common hormonal features besides hyperandrogenism that are not included in the current diagnostic criteria for PCOS. Abnormal patterns of gonadotropin secretion, including increased serum luteinizing hormone (LH) concentrations, low-normal follicle-stimulating hormone (FSH) levels, and increased $\mathrm{LH} / \mathrm{FSH}$ ratios have long been recognized as common characteristics of women with PCOS [6]. Women with PCOS have higher AMH concentrations than controls [7-10], and a high serum AMH level is regarded as a useful tool for the diagnosis of PCOS [11-13]. Both the serum AMH level $[9,14-18]$ and the $\mathrm{LH} / \mathrm{FSH}$ ratio $[19,20]$ are significantly correlated with AFC in patients with PCOS; however, the associations between these inappropriate hormonal characteristics of PCOS and the increased ovarian volume remain unresolved.

The aim of the present study was to investigate the relationship between serum gonadotropin and AMH levels in the early follicular phase and two cardinal ultrasonographic features for PCOS in Korean women with PCOS.

\section{Methods}

\section{Subjects}

This study was approved by the Institutional Review Board of Inje University Haeundae Paik Hospital. Patients aged 18-35 years who first visited Inje University Haeundae Paik Hospital for irregular menstruation and were diagnosed with PCOS between June 2010 and December 2013 were included in the study. All PCOS patients were diagnosed using the 2003 Rotterdam criteria (2 out of 3) as follows: 1 ) oligo-anovulation (menstrual cycle of > 35 days); 2) clinical and/or biochemical signs of hyperandrogenism; and 3) PCO as identified by ultrasonography, after the exclusion of other etiologies including congenital adrenal hyperplasia, androgen-secreting tumors, and Cushing's syndrome [2]. Patients who were previously diagnosed with thyroid disease or hyperprolactinemia were also excluded from the present study. Clinical hyperandrogenism was defined by the presence of hirsutism (modified Ferriman-Gallwey score >8) [21], and biochemical hyperandrogenism was defined as an elevated serum androgen level beyond the $95 \%$ confidence limits defined in controls in the study by Chae et al. [22] (total testosterone $>0.68 \mathrm{ng} /$ $\mathrm{mL}$, and/or free testosterone $>1.72 \mathrm{pg} / \mathrm{mL}$ ). PCO on ultrasonography was defined as follows: 1 ) the presence of $\geq 12$ follicles in each ovary measuring 2-9 mm in diameter; and/or 2) increased ovarian volume ( $>10 \mathrm{~mL}$ ). All participants met the ultrasonographic criteria for PCOS. Patients who had a history of previous ovarian surgery and those who had been taking oral contraceptives were excluded from the present study. Furthermore, any patient with at least one follicle with a diameter of $\geq 10 \mathrm{~mm}$ on ultrasonography was excluded from the study. Finally, 118 patients were enrolled in this retrospective study. The body mass index (BMI) was calculated by dividing the body weight $(\mathrm{kg})$ by the square of the body height $\left(\mathrm{m}^{2}\right)$.

\section{Measurement of antral follicle count and ovarian volume by ultrasonography}

Transvaginal or transrectal ultrasound examination with a Voluson S7 (General Electric Systems, Seongnam, Korea) equipped with a 7-MHz transvaginal transducer was performed in all participants in the early follicular phase between day 3 and day 4 of the menstrual cycle. For each ovary, the total number of all visible antral follicles measuring 2-9 mm in diameter was counted by continuous scanning of the entire ovary, from the inner to the outer margins in a longitudinal cross-section. The ovarian volume was calculated using the simplified formula for a prolate ellipsoid $(0.5 \times$ length $\times$ width $\times$ thickness) [2]. The total ovarian volume (TOV) was defined as the sum of the right and the left ovarian volumes, and the total antral follicle count (TFC) was defined as the sum of the right and the left AFC.

\section{Measurement of serum anti-Müllerian hormone, follicle- stimulating hormone, and luteinizing hormone levels}

Blood samples were collected from all participants in tubes without anticoagulants on the same day of ultrasonography in the early follicular phase. Sera were obtained by centrifugation for the determination of serum hormone levels. Serum hormone levels, including $\mathrm{AMH}, \mathrm{FSH}, \mathrm{LH}$, and total and free testosterone were measured using enzyme immunoassays. The serum AMH level was measured using the AMH Gen II assay (Beckman Coulter Inc., Brea, CA, USA). The serum FSH level was measured using a Roche Elecsys FSH assay (Roche, Indianapolis, IN, USA), and the serum LH level was measured using a Roche Elecsys LH assay (Roche, USA). The last menstrual period was either spontaneous or induced by the administration of medroxyprogesterone acetate (Provera, $10 \mathrm{mg} /$ day for 7 days).

\section{Statistical analysis}

All data were expressed as mean $\pm \mathrm{SD}$. All statistical analyses were performed using SPSS ver. 12.0 (SPSS Inc., Chicago, IL, USA). The correlations between serum hormone levels and ovarian morphological characteristics on ultrasonography were evaluated using Pearson's correlation coefficients and a linear regression analysis, and partial correlation coefficients were used after excluding age from the analysis. Twotailed $p$-values $<0.05$ were considered significant for all the analyses.

\section{Results}

The basal clinical and hormonal characteristics of participants are 
presented in Table 1.

The serum AMH levels were significantly correlated with the serum $\mathrm{LH}$ levels $(r=0.290, p=0.004)$ and the LH/FSH ratios $(r=0.355, p<0.001)$ (Figure $1 \mathrm{~A}$ ), but there was no significant relationship between the serum $\mathrm{AMH}$ and the FSH levels ( $p=0.081$ ).

Ultrasonography showed a highly significant correlation between TFC and TOV $(r=0.447, p<0.001)$ (Figure 1B).

As shown in Table 2 and Figure $2 \mathrm{~A}$, the serum AMH levels were significantly correlated with TFC $(r=0.515, p<0.001)$. Both the serum $\mathrm{LH}$ level and the $\mathrm{LH} / \mathrm{FSH}$ ratio were also significantly correlated with $\operatorname{TFC}(r=0.242, p=0.008$ and $r=0.250, p=0.006$, respectively), whereas the serum FSH level was not significantly correlated with TFC.

Table 1. Clinical and hormonal characteristics of participants

\begin{tabular}{lc}
\hline Characteristic & Participants $(\mathrm{n}=118)$ \\
\hline Age $(\mathrm{yr})$ & $27.00 \pm 5.67$ \\
Parity & $0.19 \pm 0.54$ \\
Body mass index $\left(\mathrm{kg} / \mathrm{m}^{2}\right)$ & $22.46 \pm 5.40$ \\
Testosterone $(\mathrm{ng} / \mathrm{mL})$ & $0.34 \pm 0.20$ \\
Free testosterone $(\mathrm{pg} / \mathrm{mL})$ & $0.72 \pm 0.73$ \\
TFC & $46.17 \pm 18.20$ \\
TOV $\left(\mathrm{cm}^{3}\right)$ & $21.13 \pm 10.24$ \\
LH $(\mathrm{IU} / \mathrm{L})$ & $10.42 \pm 6.14$ \\
FSH $(\mathrm{IU} / \mathrm{L})$ & $6.22 \pm 1.71$ \\
LH/FSH ratio & $1.71 \pm 1.02$ \\
AMH $(\mathrm{ng} / \mathrm{mL})$ & $9.86 \pm 5.44$
\end{tabular}

Values are presented as mean \pm SD.

TFC, total antral follicle count; TOV, total ovarian volume; $\mathrm{AMH}$, anti-Müllerian hormone.

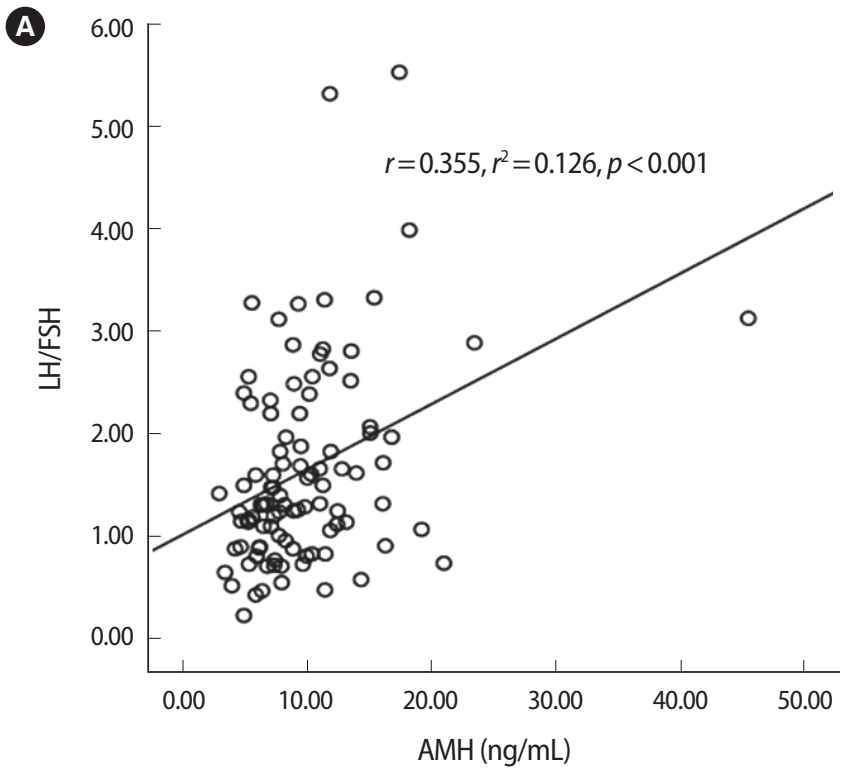

With respect to the ovarian volume, both the serum $\mathrm{LH}$ level and the $\mathrm{LH} / \mathrm{FSH}$ ratio (Figure $2 \mathrm{~B}$ ) were significantly correlated with TOV ( $r$ $=0.208, p=0.024$; and $r=0.237, p=0.010$, respectively). However, no significant association was found between the serum AMH levels and the ovarian volume $(p=0.071)$. These results did not change after adjusting for age and BMI (Table 3).

\section{Discussion}

Increased AFC ( $\geq 12$ follicles measuring 2-9 $\mathrm{mm}$ in diameter) and increased ovarian size (ovarian volume $>10 \mathrm{~cm}^{3}$ ) on ultrasonography are two cardinal features for the diagnosis of PCO [2,4]. Although PCO on ultrasonography is one of the three diagnostic criteria for PCOS, a PCO pattern on ultrasound is not an exclusive property of PCOS. PCO appearance is found in approximately $23 \%$ of women of reproductive age, whereas estimates of the incidence of PCOS vary between $5 \%$ and $10 \%$ [23]. In the present study, I found that AFC in

Table 2. Correlations between hormonal parameters and ultrasonographic volumetric parameters

\begin{tabular}{lrrrrrr} 
& \multicolumn{2}{c}{ TFC } & & \multicolumn{2}{c}{ TOV $\left(\mathrm{cm}^{3}\right)$} \\
\cline { 2 - 3 } \cline { 5 - 6 } & \multicolumn{1}{c}{$r$} & $p$ & & $r$ & $p$ \\
\hline LH (IU/L) & 0.242 & 0.008 & & 0.208 & 0.024 \\
FSH (IU/L) & -0.062 & 0.507 & & -0.036 & 0.695 \\
LH/FSH ratio & 0.250 & 0.006 & & 0.237 & 0.010 \\
AMH (ng/mL) & 0.515 & $<0.001$ & & 0.183 & 0.071 \\
\hline
\end{tabular}

TFC, total antral follicle count; TOV, total ovarian volume; r, Pearson's correlation coefficient; $\mathrm{AMH}$, anti-Müllerian hormone.

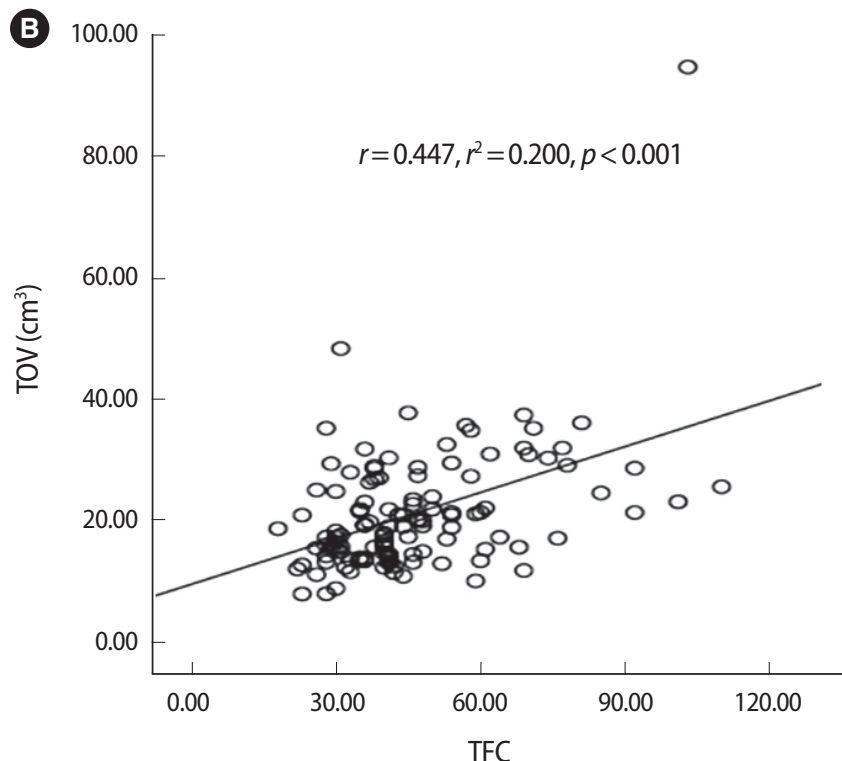

Figure 1. Correlations between (A) serum anti-Müllerian hormone levels and luteinizing hormone/follicle-stimulating hormone ratios; and (B) total antral follicle count and total ovarian volume. AMH, anti-Müllerian hormone; TFC, total antral follicle count; TOV, total ovarian volume. 

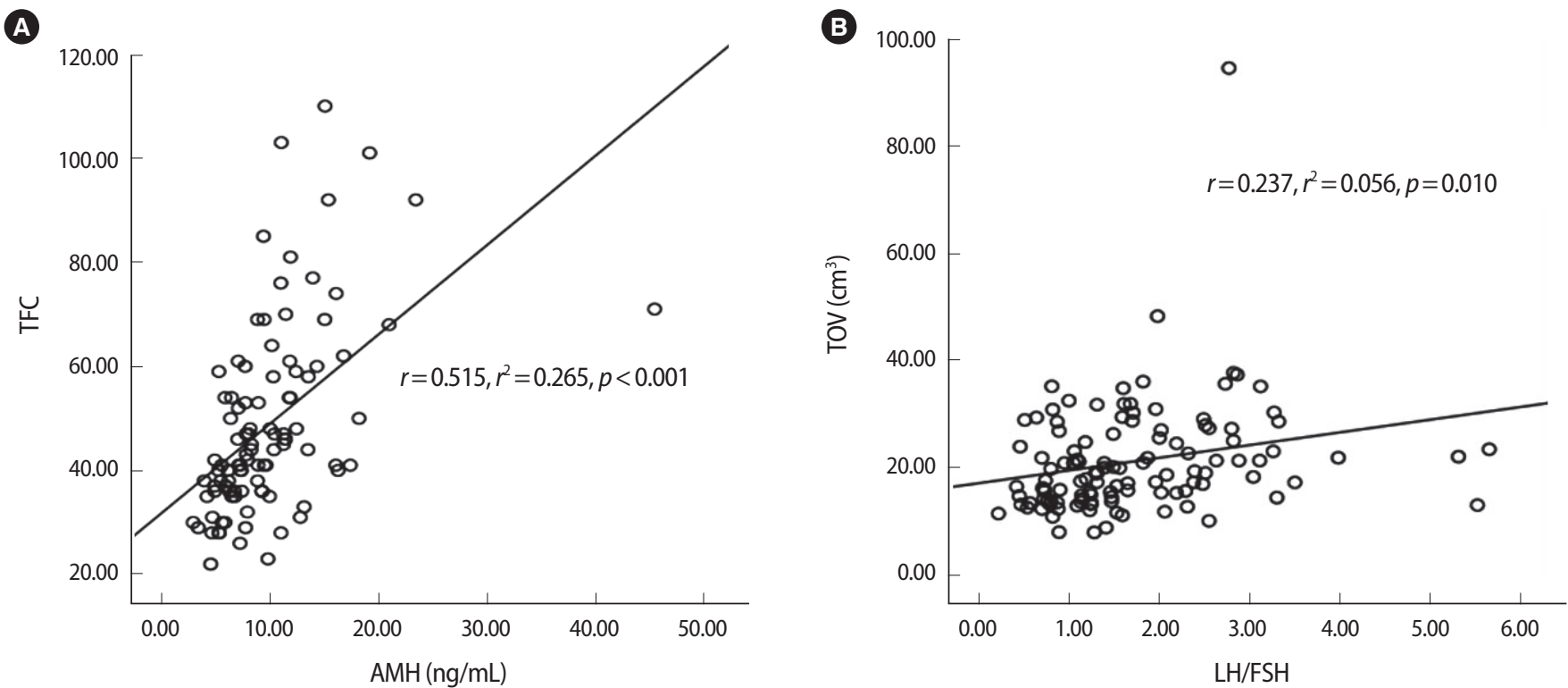

Figure 2. Correlations between (A) serum anti-Müllerian hormone levels and total antral follicle count; and (B) serum luteinizing hormone/follicle-stimulating hormone ratios and total ovarian volume. r, Pearson's correlation coefficient; TFC, total antral follicle count; TOV, total ovarian volume.

Table 3. Correlations between hormonal parameters and ultrasonographic volumetric parameters after adjustment of age and body mass index

\begin{tabular}{|c|c|c|c|c|c|c|c|c|}
\hline & \multicolumn{4}{|c|}{ TFC } & \multicolumn{4}{|c|}{ TOV } \\
\hline & $r$ & $p$ & $r^{2)}$ & $p$ & $r$ & $p$ & $r^{a)}$ & $p$ \\
\hline LH (IU/L) & 0.242 & 0.008 & 0.313 & 0.002 & 0.208 & 0.024 & 0.235 & 0.021 \\
\hline $\mathrm{FSH}(\mathrm{IU} / \mathrm{L})$ & -0.062 & 0.507 & -0.131 & 0.205 & -0.036 & 0.695 & -0.059 & 0.565 \\
\hline LH/FSH ratio & 0.250 & 0.006 & 0.343 & 0.001 & 0.237 & 0.010 & 0.267 & 0.009 \\
\hline $\mathrm{AMH}(\mathrm{ng} / \mathrm{mL})$ & 0.515 & $<0.001$ & 0.515 & $<0.001$ & 0.183 & 0.071 & 0.186 & 0.070 \\
\hline
\end{tabular}

TFC, total antral follicle count; TOV, total ovarian volume; $r$, Pearson's correlation coefficient; AMH, anti-Müllerian hormone.

a) Partial correlation coefficient adjusted by age and body mass index.

patients with PCOS was significantly correlated with the serum AMH and LH levels and the LH/FSH ratio. However, I found no significant relationship between the serum AMH level and TOV in women with PCOS, although both the serum LH level and the LH/FSH ratio were significantly correlated with TOV.

AMH is regarded as the most useful marker of the status of ovarian reserve $[7,14]$, and a highly significant relationship between the serum AMH level and AFC on ultrasonography was reported in numerous studies [9,13-18]. Women with PCOS have high AMH concentrations [8-10]. The pathogenesis of PCOS has been attributed mainly to disordered folliculogenesis causing oligo-ovulatory cycles or impaired folliculogenesis with increased preantral and small antral follicle counts, which resulted in high serum AMH levels $[24,25]$. Pigny et al. [8] reported that the AMH level was positively related to the 2-5-mm AFC as determined by ultrasonography, but not to the $6-9-\mathrm{mm}$ AFC. AMH has been proposed as a substitute for AFC in the diagnosis of PCOS $[8,11,13]$. Several cutoff values of AMH have been proposed [11-13,
25]; however, because of varying sensitivity and specificity, the optimal threshold remains unclear. The meta-analysis of the extracted data demonstrated the specificity and the sensitivity in diagnosing PCOS in the symptomatic women of $79.4 \%$ and $82.8 \%$, respectively, for a cutoff value of AMH of $4.7 \mathrm{ng} / \mathrm{mL}$ [13].

In the present study, the serum AMH level was highly correlated with AFC, and the correlation between the AMH level and TFC ( $r=$ $0.515)$ was higher than that between the LH level and TFC ( $r=0.242)$ and that between the LH/FSH ratio and TFC $(r=0.250)$. My results were in agreement with those reported by Fanchin et al. [15], who showed that the serum AMH levels were more robustly correlated with the number of early antral follicles than inhibin B, E2, FSH, and LH on day 3 of the menstrual cycle. In contrast, I found no significant correlation between the serum AMH level and the ovarian volume, and this result is in disagreement with that of a previous study [26] showing that the AMH levels were positively correlated with the ovarian volume $(r=0.222, p=0.027)$. 
Inappropriate secretion of $\mathrm{LH}$ and an abnormal LH/FSH ratio are the main factors associated with the continuation of the anovulatory state in PCOS subjects. Although abnormal LH secretion is not included among the current Rotterdam diagnostic criteria for PCOS [2], it is included among the diagnostic criteria proposed in Japan (JSOG) [27]. The LH/FSH ratio is a valuable diagnostic tool for the evaluation of women with PCOS and oligomenorrhea or anovulation [28], and inappropriate $\mathrm{LH}$ secretion is inversely related to $\mathrm{BMI}$ and percentage body fat in a continuous fashion [19,29]. However, because the measurement of gonadotropin levels in women with PCOS has produced varied results under different conditions, the role of the $\mathrm{LH} / \mathrm{FSH}$ ratio in identifying women with PCOS remains controversial [30,31]. Some authors have reported that the $\mathrm{LH} / \mathrm{FSH}$ ratios are significantly related to AFC $[19,20]$ and the ovarian volume $[19,32]$. In our study, the serum $\mathrm{LH}$ level and the $\mathrm{LH} / \mathrm{FSH}$ ratio were significantly correlated with both AFC and the ovarian volume in women with PCOS, which is consistent with the results of a previous study [19].

The two main histological features of PCO are an excessive number of follicles, also termed as multifollicularity, and stromal hypertrophy [3]. Although AFC and the ovarian volume were significantly correlated with each other in the present study, the main determinant of ovarian enlargement in women with PCOS is the volume of the ovarian stroma. Increased stromal echogenicity and/or stromal volume are specific to PCO $[3,5]$, and the ovarian stromal area and volume are significantly correlated with the levels of androstenedione [33,34]. At present, neither a qualitative nor a quantitative assessment of the ovarian stroma is required to define PCO because the measurement of the ovarian volume is a good surrogate for the quantification of stroma in clinical practice $[2,4]$. The ovarian volume can be more easily and reliably measured in routine practice than the ovarian stroma.

The present study showed a positive correlation between the serum $\mathrm{AMH}$ level and AFC in women with PCOS; however, the serum $\mathrm{AMH}$ level was not significantly related to the ovarian volume on ultrasonography. One possible explanation is that the ovarian stromal volume is not significantly affected by the serum AMH level. You et al. [26] reported that the AMH levels are significantly correlated with the ovarian volume in Korean women with PCOS, and these results are contrary to the findings of the present study. However, in the study by You et al. [26], the correlation coefficient between the $\mathrm{AMH}$ level and the ovarian volume $(r=0.222)$ was smaller than that between the AMH level and AFC ( $r=0.535)$, and it is possible that the correlation between the $\mathrm{AMH}$ level and the ovarian volume is mainly influenced by the positive effect of AMH on AFC and not on the ovarian stroma in women with PCOS.

The present study has limitations that mostly stem from its retrospective study design and relatively small sample size. This study was a preliminary study, and I did not investigate other ultrasonographic parameters including ovarian stromal echogenicity and ovarian arterial blood flow indices.

In conclusion, the present study showed that the serum LH level and the $\mathrm{LH} / \mathrm{FSH}$ ratio were significantly correlated with the ovarian volume, whereas the serum AMH level was not related to the ovarian volume in Korean women with PCOS. My results suggest that the serum $\mathrm{LH}$ level and the LH/FSH ratio are more useful for representing the status of the ovarian volume than the serum AMH level in women with PCOS. Further prospective large-scale trials are needed to confirm this preliminary finding.

\section{Conflict of interest}

No potential conflict of interest relevant to this article was reported.

\section{References}

1. Kuzbari O, Doralis J, Peterson CM. Endocrine disorders. In: Berek JS, editor. Berek \& Novak's gynecology. 15th ed. Philadelphia: Lippincott Williams \& Wilkins; 2012. p. 1075-80.

2. Rotterdam ESHRE/ASRM-Sponsored PCOS Consensus Workshop Group. Revised 2003 consensus on diagnostic criteria and longterm health risks related to polycystic ovary syndrome. Fertil Steril 2004;81:19-25.

3. Jonard S, Robert Y, Cortet-Rudelli C, Pigny P, Decanter C, Dewailly D. Ultrasound examination of polycystic ovaries: is it worth counting the follicles? Hum Reprod 2003;18:598-603.

4. Balen AH, Laven JS, Tan SL, Dewailly D. Ultrasound assessment of the polycystic ovary: international consensus definitions. Hum Reprod Update 2003;9:505-14.

5. Buckett WM, Bouzayen R, Watkin KL, Tulandi T, Tan SL. Ovarian stromal echogenicity in women with normal and polycystic ovaries. Hum Reprod 1999;14:618-21.

6. Fritz MA, Speroff $L$, editors. Clinical gynecologic endocrinology and infertility. 8th ed. Philadelphia: Lippincott Williams \& Wilkins; 2011. p. 501-18.

7. Lee JR, Kim SH. Anti-Mullerian hormone and female reproduction. Korean J Obstet Gynecol 2009;52:285-300.

8. Pigny P, Merlen E, Robert Y, Cortet-Rudelli C, Decanter C, Jonard S, et al. Elevated serum level of anti-mullerian hormone in patients with polycystic ovary syndrome: relationship to the ovarian follicle excess and to the follicular arrest. J Clin Endocrinol Metab 2003;88:5957-62.

9. Laven JS, Mulders AG, Visser JA, Themmen AP, De Jong FH, Fauser BC. Anti-Mullerian hormone serum concentrations in normoovulatory and anovulatory women of reproductive age. J Clin Endocrinol Metab 2004;89:318-23. 
10. La Marca A, Orvieto R, Giulini S, Jasonni VM, Volpe A, De Leo V. Mullerian-inhibiting substance in women with polycystic ovary syndrome: relationship with hormonal and metabolic characteristics. Fertil Steril 2004;82:970-2.

11. Dewailly D, Gronier H, Poncelet E, Robin G, Leroy M, Pigny $P$, et al. Diagnosis of polycystic ovary syndrome (PCOS): revisiting the threshold values of follicle count on ultrasound and of the serum AMH level for the definition of polycystic ovaries. Hum Reprod 2011;26:3123-9.

12. Eilertsen TB, Vanky E, Carlsen SM. Anti-Mullerian hormone in the diagnosis of polycystic ovary syndrome: can morphologic description be replaced? Hum Reprod 2012;27:2494-502.

13. Iliodromiti S, Kelsey TW, Anderson RA, Nelson SM. Can anti-Mullerian hormone predict the diagnosis of polycystic ovary syndrome? A systematic review and meta-analysis of extracted data. J Clin Endocrinol Metab 2013;98:3332-40.

14. van Rooij IA, Broekmans FJ, Scheffer GJ, Looman CW, Habbema $J \mathrm{D}$, de Jong $\mathrm{FH}$, et al. Serum antimullerian hormone levels best reflect the reproductive decline with age in normal women with proven fertility: a longitudinal study. Fertil Steril 2005;83:979-87.

15. Fanchin R, Schonauer LM, Righini C, Guibourdenche J, Frydman $\mathrm{R}$, Taieb J. Serum anti-Mullerian hormone is more strongly related to ovarian follicular status than serum inhibin B, estradiol, FSH and LH on day 3. Hum Reprod 2003;18:323-7.

16. Homburg R, Ray A, Bhide P, Gudi A, Shah A, Timms P, et al. The relationship of serum anti-Mullerian hormone with polycystic ovarian morphology and polycystic ovary syndrome: a prospective cohort study. Hum Reprod 2013;28:1077-83.

17. Nardo LG, Christodoulou D, Gould D, Roberts SA, Fitzgerald CT, Laing I. Anti-Mullerian hormone levels and antral follicle count in women enrolled in in vitro fertilization cycles: relationship to lifestyle factors, chronological age and reproductive history. Gynecol Endocrinol 2007;23:486-93.

18. de Vet A, Laven JS, de Jong FH, Themmen AP, Fauser BC. Antimullerian hormone serum levels: a putative marker for ovarian aging. Fertil Steril 2002;77:357-62.

19. Shim AR, Hwang Yl, Lim KJ, Choi YM, Jeon YE, Seo SK, et al. Inappropriate gonadotropin secretion in polycystic ovary syndrome: the relationship with clinical, hormonal and metabolic characteristics. Korean J Obstet Gynecol 2011;54:659-65.

20. Wiser A, Shehata F, Holzer H, Hyman JH, Shalom-Paz E, Son WY, et al. Effect of high $\mathrm{LH} / \mathrm{FSH}$ ratio on women with polycystic ovary syndrome undergoing in vitro maturation treatment. J Reprod
Med 2013;58:219-23.

21. Hatch R, Rosenfield RL, Kim MH, Tredway D. Hirsutism: implications, etiology, and management. Am J Obstet Gynecol 1981; 140:815-30.

22. Chae SJ, Kim JJ, Choi YM, Hwang KR, Jee BC, Ku SY, et al. Clinical and biochemical characteristics of polycystic ovary syndrome in Korean women. Hum Reprod 2008;23:1924-31.

23. Polson DW, Adams J, Wadsworth J, Franks S. Polycystic ovaries: a common finding in normal women. Lancet 1988;1:870-2.

24. Visser JA, Themmen AP. Anti-Mullerian hormone and folliculogenesis. Mol Cell Endocrinol 2005;234:81-6.

25. Sahmay S, Atakul N, Aydogan B, Aydin Y, Imamoglu M, Seyisoglu $H$. Elevated serum levels of anti-Mullerian hormone can be introduced as a new diagnostic marker for polycystic ovary syndrome. Acta Obstet Gynecol Scand 2013;92:1369-74.

26. You SY, Park SY, Yang GY, Jeong KA, Kim YJ, Chung HW. Anti-Mullerian hormone in women with polycystic ovary syndrome. Korean J Obstet Gynecol 2012;55:315-24.

27. Kubota T. Update in polycystic ovary syndrome: new criteria of diagnosis and treatment in Japan. Reprod Med Biol 2013;12:71-7.

28. Hsu MI, Liou TH, Liang SJ, Su HW, Wu CH, Hsu CS. Inappropriate gonadotropin secretion in polycystic ovary syndrome. Fertil Steril 2009;91:1168-74.

29. Arroyo A, Laughlin GA, Morales AJ, Yen SS. Inappropriate gonadotropin secretion in polycystic ovary syndrome: influence of adiposity. J Clin Endocrinol Metab 1997;82:3728-33.

30. Turhan NO, Toppare MF, Seckin NC, Dilmen G. The predictive power of endocrine tests for the diagnosis of polycystic ovaries in women with oligoamenorrhea. Gynecol Obstet Invest 1999;48:183-6.

31. Cho LW, Jayagopal V, Kilpatrick ES, Holding S, Atkin SL. The LH/ FSH ratio has little use in diagnosing polycystic ovarian syndrome. Ann Clin Biochem 2006;43:217-9.

32. Sikka P, Gainder S, Dhaliwal LK, Bagga R, Sialy R, Sahdev S. Ultrasonography of the ovaries and its correlation with clinical and endocrine parameters in infertile women with PCOS. Int J Fertil Womens Med 2007;52:41-7.

33. Kyei-Mensah AA, LinTan S, Zaidi J, Jacobs HS. Relationship of ovarian stromal volume to serum androgen concentrations in patients with polycystic ovary syndrome. Hum Reprod 1998;13:1437-41.

34. Dewailly D, Robert Y, Helin I, Ardaens Y, Thomas-Desrousseaux P, Lemaitre $L$, et al. Ovarian stromal hypertrophy in hyperandrogenic women. Clin Endocrinol (Oxf) 1994;41:557-62. 